\title{
Students' performance on missing-value word problems: a cross-national developmental study
}

\begin{abstract}
This study investigates Spanish and Chinese students' performance on both addition problems and proportion problems considering a cross-national perspective. The effect of number structure and nature of quantities was also considered. Nine hundred twenty-five 4th to 8th graders (453 Chinese, 472 Spanish) took a test which is composed of addition and proportion problems in a missing-value format. The findings showed that Chinese students held a different developmental model of the additive and proportional reasoning from Spanish students. Moreover, number structure and nature of quantities had a different impact in each country. These results indicated that students' additive and proportional reasoning may vary from country to country due to the different cultures and educational environments. Further studies with a larger-scale sample will be needed to confirm such cross-national effects.
\end{abstract}

\section{Keywords}

Additive reasoning $\cdot$ Proportional reasoning $\cdot$ Cross-national study $\cdot$ Overuse of proportionality

\section{Introduction}

Thinking mathematically is the principal aim of mathematical teaching and learning. Students start thinking additively in the early years of elementary school. Then, after they enter higher grades, multiplicative thinking is introduced to their developmental pathway. It is a great achievement for students to transform from additive thinking to multiplicative thinking; however, this transition is not easy (Harel and Confrey 1994; Nesher 1988; Verschaffel et al. 2007). Many students are difficult to distinguish the difference between additive and multiplicative contexts (De Bock et al. 2007; Fernández and Llinares 2012; Van Dooren et al. 2008). For example, for the following word problems:

\footnotetext{
"Stephanie and Jane are washing plates. They wash equally fast but Jane started earlier. When Stephanie has washed 4 plates, Jane has washes 12 plates. If Stephanie has washed 20 plates, how many plates has Jane washed?"

"Stephanie and Jane are washing plates. They started together but Jane washes faster. When Stephanie has washed 4 plates, Jane has washes 12 plates. If Stephanie has washed 20 plates, how many plates has Jane washed?"
}

Both word problems have the same format (four quantities are involved but the last one is not given) and refer to a co-variation situation; however, in the first problem, the given numbers are linked additively and should be solved by considering an additive relationship (Jane has washed $12-4=8$ plates more than Stephanie). While in the second problem, the given numbers are related proportionally (or multiplicatively), and to solve this problem, a proportional relationship (Jane has washed 12: $4=3$ times more plates than Stephanie) should be used.

Many researchers have shown that students tend to overlook the relationship between the 
given numbers when they are solving such problems: Some students are very good at solving addition problems, but they tend to use addition to dealing with proportional situations (Hart et al. 1981; Tourniaire and Pulos 1985); some students perform well in proportion problems, but they treat addition problems as proportional ones, and such phenomenon had been called the overuse of proportionality (Li et al. 2014; Misailidou and Williams 2003; Van Dooren et al. 2009). Later studies indicated that students' overuse of additive and proportional methods is highly related to the development of correspondent reasoning ability. These researchers also observed different developmental patterns of students' additive and proportional reasoning in different countries (Fernández et al. 2012; Van Dooren et al. 2010). The results of these studies suggested that students' mathematical performance may vary by countries due to their different teaching and learning environments. This would be an interesting explanation as we can see how different cultural and pedagogical systems affect students' mathematical thinking.

However, previous studies did not use the same experimental materials and procedurals and it is hard to conclude that such differences are caused by national differences. Thus, to answer the question about whether students' additive and proportional reasoning varies from country to country, a cross-national study that conducts the same experiment operation is needed. In addition, previous studies mostly focus on the development of students' overuse of proportional reasoning, and there are few studies that have investigated the phenomenon of the overuse of additive reasoning in the transition from elementary to secondary school. It might be interesting to investigate how students will overuse additive methods in countries with different curricular traditions. Thus, this study aims at comparing students' performance on both addition and proportion problems in a cross-national perspective.

\section{Theoretical and empirical background}

\section{A cross-national perspective}

Cross-national studies provide us special opportunities to understand how different cultures and educational environments affect students' mathematical achievement. Based on the results of these researches, we can design intervention programs to improve students' learning (Cai 2004; Robitaille and Travers 1992). Some international studies of students' mathematic performance have received considerable attention by the educational researchers as well as by the general public (such as TIMSS, OECD). To some extent, these studies reveal the current situation of international mathematic education and the strength and weakness of mathematics education in different countries (Mullis et al. 2008; Mullis et al. 2012; OECD 2010). However, some researchers pointed out that discussing cross-national performance has focused too much on international ranking. It could be more illuminating if we were to probe students' thinking and reasoning to better understand how they approach mathematical problem solving (Cai 2000; Jiang et al. 2014). Therefore, by investigating how students solve addition and proportion problems in different countries, we have an opportunity to find out how different cultures or instructional systems affect students' additive and proportional reasoning. This is critical for the improvement of mathematical teaching and learning.

According to previous studies, the trends in students' performance on both addition and proportion problems along grade levels may differ from country to country. For example, Van Dooren et al. (2010) found that for Flemish students, the overuse of additive methods decrease from 3rd to 6th grade and, at the same time, the overuse of proportional methods increase during this period. Such results indicated that students had a tendency to apply additive methods without considering the situations in the lower grades of elementary school and to apply proportional methods in the same way in the upper grades of elementary school. Later 
study confirm such findings with Spanish students when considering a larger range of age: from 3rd grade to 12th grade, however, this study suggested that the turning point of students' development of proportional reasoning may vary from Spain to Belgium (Fernández et al. 2012). Li et al. (2014) found that all Chinese 5th to 8th graders performed better on proportion problems than they did on addition problems, but they also showed the overuse of proportionality on addition problems. Furthermore, Li et al. (2014) also found that 6th graders had the strongest tendency to overuse proportional methods and that this tendency decreased from 7 th grade onward. Unlike the studies with Flemish and Spanish students, Li et al.'s study observed a decrease in the overuse of proportional methods.

These results indicated that different cultural and educational environments may affect students' development of additive and proportional reasoning. However, the studies referenced above did not use the same experiment operation, so we cannot be sure that such differences were caused by national difference. Thus, to identify the cross-national effect on students' additive and proportional reasoning, the present study used the same materials and chose Chinese and Spanish students as our subjects. The reasons we chose these two countries are as follows:

First, the mathematical performances of Chinese students and Spanish students are different. According to previous studies, Chinese students' mathematical performance in TIMSS is better than Spanish students' (TIMSS, 1995, 2011). However, it is unclear whether or not highperformance students would use additive and proportional methods in a better way. Instead, high performance in additive or proportional problem-solving may lead to a high level of the overuse of the relevant methods (Van Dooren et al. 2010). Thus, a worthy objective would be to identify the different reasoning models held by the high-performance group and the lowperformance group.

Second, the mathematics learning environments are different in China and Spain. In elementary school, part of the curricula settings in China and Spain are similar, as both Chinese and Spanish students learn the basic mathematical concepts at the same grade level (addition and subtraction: 1st grade; multiplication and division: 2nd grade; fraction, decimals, and percentage: 3rd-5th grade; concepts of ratio and proportion: 5th-6th grade). However, Chinese 6th graders also learn direct and inverse proportionality and the "rule of three" (i.e., "cross-multiplication") while such learning occurs in 7th-8th grade in Spain. For both countries, students are confronted with missing-value problems from 1st grade through 6th grade-most problems are in additive situations in the early years while proportional ones increase from 3rd grade onward. However, proportion problems become dominate in 6th grade in China while such phenomenon appears in secondary school in Spain and missing-value problems can hardly be found in Chinese secondary school at all. (Beijing Normal University Press (BNUP) 2013; RD 112/2007; RD 108/2014). Furthermore, Chinese students usually do more practice and homework, and the classroom instruction is often content-based, examination-driven, and teacher-centered (Fan et al. 2004; Siegler and Mu 2008). Instruction is usually conducted in a whole-classroom setting, with a large class of 50-60 students and with little interaction among the students. While in Spain, classroom instruction is usually conducted in small classes (20-30 students in a class). Such differences may also affect students' mathematical thinking development.

Third, previous Chinese and Spanish studies provided a stronger foundation to understand the developmental pattern of students' additive and proportional reasoning because both studies considered an age range that covered elementary and secondary school (Fernández et al. 2011, 2012; Li et al. 2014). Most especially, the studies provided information about how students used additive and proportional methods when they started to learn higher-order mathematical knowledge (e.g., algebra or binary equations) in secondary school. Furthermore, 
these studies indicated that the developmental trends of students' use of additive and proportional solutions may be different in China and Spain: first, Chinese students reached the peak of using the proportional method earlier than Spanish students; second, Chinese secondary school students used less proportional methods in addition problems. This difference may be caused by national differences, and it would be interesting to investigate elementary and secondary students simultaneously in China and Spain to see if cultural factors would affect students' additive and proportional reasoning.

\section{Other factors that affect students' tendency to apply additive and proportional methods in missing-value word problems}

It is well documented that the ratio of the numbers in the problem affects students' solutions to missing-value word problems (Fernández et al. 2009; Kaput and West 1994; Van Dooren et al. 2009). Researchers found that when integer ratios were involved in missing-value problems (e.g., the given numbers were 3,12, and 6, the fourth is unknown), students performed better on proportion problems, but at the meantime, they also overused proportional solutions to nonproportion problems. Inversely, students had a stronger tendency to use additive methods in both addition and proportion problems when non-integer ratios were involved. The effect of such number structure was confirmed by many studies (Fernández et al. 2009, 2011, 2012; Li et al. 2014). However, Fernández et al. (2012) found that number structure affects all the Spanish students from 4th to 12th grade; for Chinese students, the ratios affect 5th and 6th graders' use of proportional methods while 7th and 8th graders were not influenced. It is possible that such effects would differ from country to country. Thus, the present study also investigates how number structure may affect students' additive and proportional reasoning through a cross-national perspective.

The nature of the quantities is another variable that affects students' solution to addition and proportion problems. Many studies indicated that discrete and continuous quantities (e.g., quantity of boxes and quantity of skating distance) affect students' strategies of problem solving (Boyer et al. 2008; Jeong et al. 2007; Tourniaire and Pulos 1985). In particular, Fernández et al.'s (2012) study indicated that nature of quantities had a low impact on the tendency of using proportional methods. Students had a slightly stronger tendency to apply proportional method in the discrete context than in the continuous context, independent of whether the problems were proportional or non-proportional. However, results on the impact of this variable are scarce and more research is needed. Therefore, we also consider the effect of nature of quantities and the possible interaction between this variable and national difference.

\section{Research questions}

In this study, we investigated simultaneously the developmental trajectory of additive and proportional reasoning in Spanish and Chinese 4th to 8th grade students while taking into account a cross-national perspective. The research questions are as follows:

How does Chinese and Spanish students' additive and proportional reasoning develop from 4th grade to 8th grade? Are there differences between the developmental models of Chinese and Spanish students?

How do number structure and the nature of quantities affect students' additive and proportional reasoning? Do these two variables interact with national difference? 


\section{Method}

\section{Participants and context}

Participating in this study were 925 elementary and secondary students, including 453 Chinese students (102 4th graders, 100 5th graders, 101 6th graders, 90 7th graders, and 60 8th graders) and 472 Spanish students (65 4th graders, 68 5th graders, 64 6th graders, 124 7th graders, and 151 8th graders). Participating schools were two Chinese elementary schools, two Spanish elementary schools, one Chinese secondary school, and two Spanish secondary schools. These schools were from different cities in Spain and from one city in China.

In the Chinese context, students in elementary and secondary school are assigned to the nearest school districts and streaming students based on ability is forbidden by the government. In Spain, students are randomly assigned into different school districts. In both countries, all the schools were from typical school districts and they participated voluntarily. To control for students' mathematical abilities, neither outstanding schools nor inferior schools were considered. We also randomly selected classes of each grade of our participating schools. All the participants were from mixed socioeconomic backgrounds.

\section{Instrument and procedure}

The instrument and the procedure were the same in China and Spain. The original version of the word problems test was in Spanish (Fernández et al. 2011, 2012), while in China, it was translated into Chinese.

Table 1 shows the design of the problems. We designed four addition problem and four proportion problems by manipulating the second sentence (e.g., "They wash equally fast but Jane started earlier" in the addition problem and "They started together but Jane washes faster" in the proportion problem). Half of the addition and proportion problems refer to a situation with discrete quantity (e.g., the number of plates) and half of them refer to a situation with continuous quantity (e.g., the amount of the running distance). For the given numbers in the problem, we also created integer and the non-integer ratios (e.g., 24:12 and 48:12 in A-D-I; 18:12 and 30:12 in A-D-N in Table 1).

To avoid effects of practice or stereotyped responses, four buffer problems were included in the test. Thus, the full test consisted of 12 problems (eight experimental problems and four buffer problems), and this was the same for all grades. By varying the sequence of the 12 problems, we created eight different versions of the test. Note that these versions were identical except the order of the problems differed. Furthermore, all experimental problems were controlled for number size (numbers with 1 to 3 digits), calculation complexity (the correct answer is always an integer number), the context (always actions), and the position of the unknown quantity (always in the last position).

The eight test versions were distributed randomly to the students. The students had 40 to $50 \mathrm{~min}$ (i.e., the regular amount of time for a math class) to complete the test. Students were asked to write down the calculation process and were allowed to use calculators.

Table 1 Examples of additive (A) and proportional (P) problems with manipulation of the number structure (integer ratios between given numbers $[\mathrm{I}]$ and non-integer ratios $[\mathrm{N}]$ ) and the nature of quantities (discrete variable [D] and continuous variables [C]) (Fernández et al. 2012) 
A-D Stephanie and Jane are washing plates. They wash equally fast but Jane started earlier. When Stephanie has washed 12 plates, Jane has washed 24 plates. If Stephanie has washed 48 plates, how many plates has Jane washed?

P-D Stephanie and Jane are washing plates. They started together but Jane washes faster. When Stephanie has washed 12 plates, Jane has washed 24 plates. If Stephanie has washed 48 plates, how many plates has Jane washed?

A-C Ann and Lily are running. They run equally fast but Lily started earlier. When Ann has run $150 \mathrm{~m}$, Lily has run $300 \mathrm{~m}$. If Ann has run $600 \mathrm{~m}$, how many meters has Lily run?

P-C Ann and Lily are running. They started together but Lily runs faster. When Ann has run $150 \mathrm{~m}$, Lily has run $300 \mathrm{~m}$. If Ann has run $600 \mathrm{~m}$, how many meters has Lily run?
Stephanie and Jane are washing plates. They wash equally fast but Jane started earlier. When Stephanie has washed 12 plates, Jane has washed 18 plates. If Stephanie has washed 30 plates, how many plates has Jane washed?

Stephanie and Jane are washing plates. They started together but Jane washes faster. When Stephanie has washed 12 plates, Jane has washed 18 plates. If Stephanie has washed 30 plates, how many plates has Jane washed?

Ann and Lily are running. They run equally fast but Lily started earlier. When Ann has run $80 \mathrm{~m}$, Lily has run $120 \mathrm{~m}$. If Ann has run 200 m, how many meters has Lily run?

Ann and Lily are running. They started together but Lily runs faster. When Ann has run $80 \mathrm{~m}$, Lily has run $120 \mathrm{~m}$. If Ann has run $200 \mathrm{~m}$, how many meters has Lily run? 


\section{Results}

In this section, we first present the development of proportional and additive methods from 4th grade to 8th grade for addition and proportion problem in China and Spain. We then show the influence of number structure and nature of quantities on the development of students' use of proportional and additive methods and how such influence differs from China to Spain. Note that we could discuss all the results of the analyses, however, since the present study is a crossnational study, we will mainly discuss the effect of the "country" variable and its interaction effects with other variables.

\section{The development of the use of additive methods in China and Spain}

According to the results of the repeated measures logistic regression analysis on additive answers, the main effect of "country" was not significant, $X^{2}(1, N=925)=0.68, p=0.435$, but the interaction effect of "country" $\times$ "grade" was significant, $X^{2}(4, N=925)=15.060$, $p=0.005$, and there was a significant interaction effect of "country" $\times$ "type of problem", $X^{2}(1, N=925)=54.576, p<0.001$. Moreover, the interaction effect of "country" $x$ "grade" $\times$ "type of problem" was significant, $X^{2}(4, N=925)=18.522, p=0.001$. Further analyses showed that for Chinese students, the interaction effect of "grade" $\times$ "type of problem" was significant, $X^{2}(4, N=453)=31.578, p<0.001$, while for Spanish students, such an effect was not significant, $X^{2}(4, N=472)=7.061, p=0.130$. Such results indicate that for Chinese students, the use of additive methods along grades varies with the type of problem, while for Spanish students, the developmental tracks of the use of additive methods on the two types of problems are similar. As we can see in Figs. 1 and 2, for Chinese students, the percentages of additive answers to addition problems remained at a high level during 4th grade to 8th grade, but such percentages in proportion problems apparently decreased during this period. For Spanish students, however, though the percentages of additive answers to addition problems remained at a high level, similar to the Chinese students, their additive answers to proportion problems did not show an obvious decrease during 4th grade to 8th grade.

For addition problems, pairwise comparisons showed that the differences of using additive methods were significant in 4th grade and 5th grade between Chinese and Spanish students. Chinese 4th graders and 5th graders significantly used more additive methods on addition problems, while in other grades, such differences were not significant. For proportion problems, the differences of students' use of additive methods were significant in the all the grades between China and Spain except for the 5th grade. Compared to their Spanish peers, Chinese 4th graders significantly used more additive methods on proportion problems, while 6th graders, 7th graders, and 8th graders did just the reverse (see Fig. 2). Moreover, for Chinese students, the differences of the percentage of additive answers between addition problems and proportion problems were significant in all grades except grade 5, while for Spanish students, such differences were only significant in grade $8 .{ }^{1}$

These data indicate that Chinese students in lower grades tend to use more additive methods on addition problems than their Spanish peers, and their tendency to overuse additive methods on proportion problems is greater. On the other hand, most Chinese and Spanish students in the higher grades could correctly solve addition problems, but Spanish students in the higher grades incorrectly use more additive methods on proportion problems than Chinese students. Therefore, although Chinese students in the lower grades tend to struggle in proportion problems (using additive methods), this tendency starts to significantly decrease in the 6th grade. 


\section{The development of the use of proportional methods in China and Spain}

For the occurrence of proportional answers, the repeated measures logistic regression analysis showed that the main effect of "country" was significant, $X^{2}(1, N=925)=82.700, p<0.001$, and there was a significant "country" $\times$ "grade" interaction effect, $X^{2}(4, N=925)=10.120$, $p=0.038$ and a significant interaction effect of "country" $\times$ "type of problem", $x^{2}(1$, $\mathrm{N}=925)=7.239, \mathrm{p}=0.007$. It is important to note that we also had a significant interaction effect of "country" $\times$ "grade" $\times$ "type of problem", $x^{2}(4, N=925)=11.912, p=0.018$. Further analyses showed that for Chinese students, the interaction effect of "grade" $\times$ "type of problem" was significant, $X^{2}(4, N=453)=16.532, p=0.002$, while for Spanish students, such an effect was not significant, $X^{2}(4, N=472)=1.878, p=0.758$. According to the results, we can see that for Chinese students, their development of using proportional methods varied with the type of problem, while Spanish students showed similar developmental patterns on both types of problems. As we can see in Figs. 3 and 4, for Chinese students, the percentages of proportional answers to proportion problems distinctly increased during 4th grade to 8th grade,

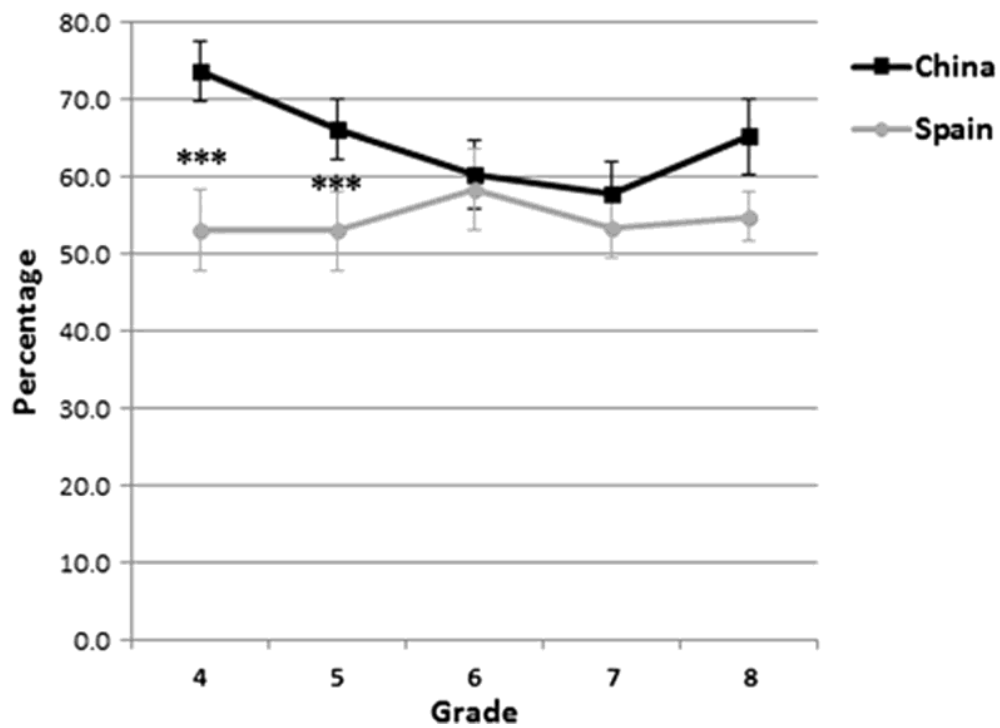

Fig. 1 The development of additive methods on additive problems from 4th grade to 8th grade in China and Spain. Asterisk refers to the result of pairwise comparison between China and Spain in each grade and has significant difference (the same as Figs. 2, 3, and 4)

and such a tendency was especially significant between 5th grade and 6th grade (with the introduction in the curricula of procedure of "cross-multiplication"). On the other hand, the percentages of using proportional methods to solve addition problems remained at a medium level during 4th grade to 8th grade, though there was a small leap between 5th grade and 6th grade. For Spanish students, the percentages of the use of proportional methods on both proportion problems and addition problems increased slowly during 4th grade to 7 th grade and had an obvious increase between 7 th grade and 8th grade, as we have mentioned above, although the developmental trends were almost the same.

For proportion problems, further pairwise comparisons showed that the differences of the use of proportional methods were significant in all the grades between Chinese and Spanish students. Chinese students used proportional methods on proportion problems significantly more often than Spanish students. For addition problems, the differences of students' use of 
proportional methods were significant in all grades between China and Spain except the 8th grade. Compared to Spanish students, Chinese students used erroneous proportional methods on addition problems significantly more often during 4th grade to 7 th grade, while 8 th graders in both countries showed no significant differences. Such results indicate that Chinese students perform better in solving proportion problems than Spanish students, but they also have a greater tendency to overuse proportional methods on addition problems than Spanish students.

Note that for Chinese students, the differences between the percentages of the proper use and the overuse of the proportional method were significant in all grades, while for Spanish students, such differences were not significant except for grade $8 .^{2}$

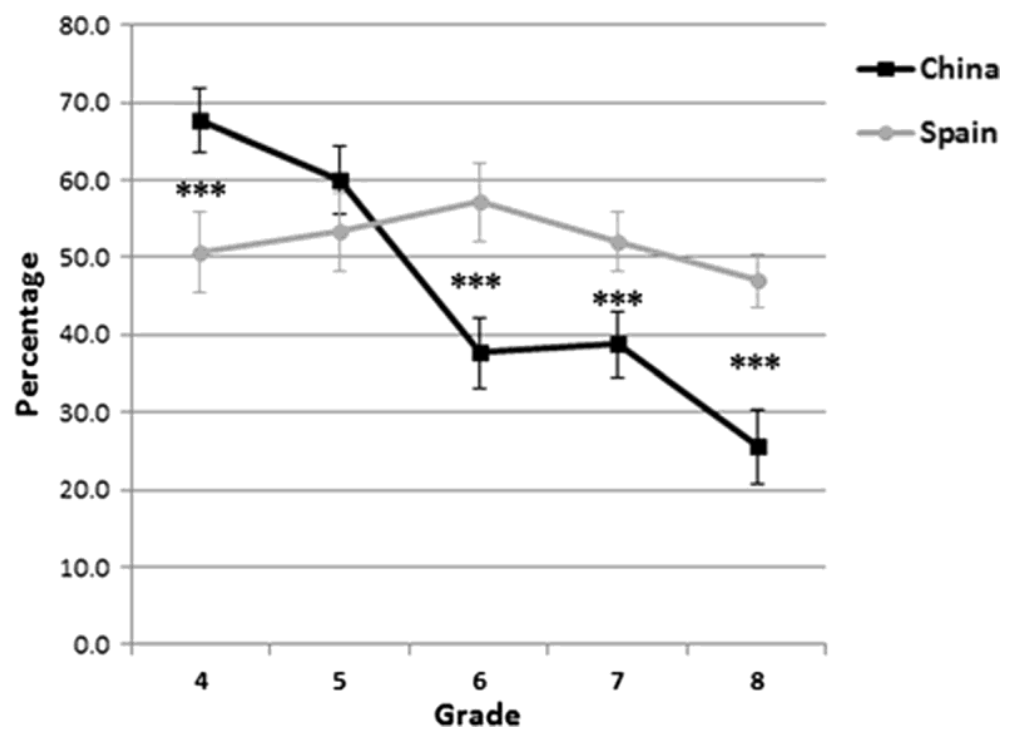

Fig. 2 The development of additive methods on proportional problems from 4th grade to 8th grade in China and Spain

\section{The effect of number structure and its interaction with country}

Tables 2 and 3 present the results of the effect of number structure on students' use of additive and proportional methods in two types of problem along grade levels in China and Spain.

For the use of additive methods, results showed that the main effect of "number structure" was significant, $X^{2}(1, N=925)=91.844, p<0.001$; also, the interaction effect of "grade" $\times$ 


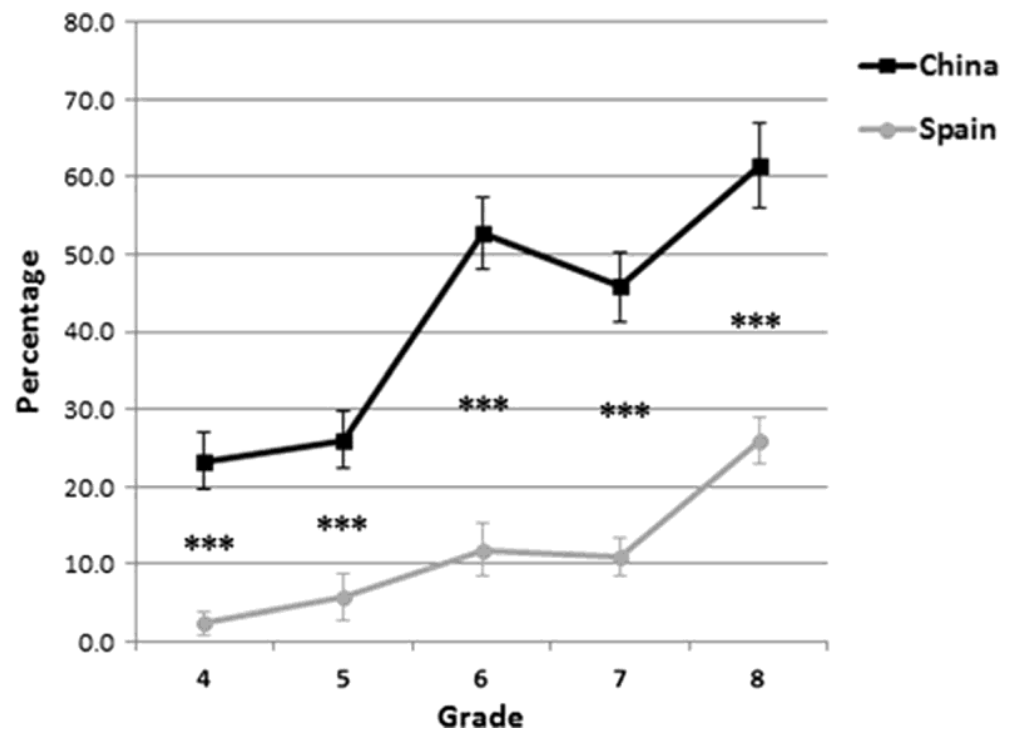

Fig. 3 Thedevelopment of proportional methods on proportional problems from 4th grade to 8th grade in Chinaand Spain

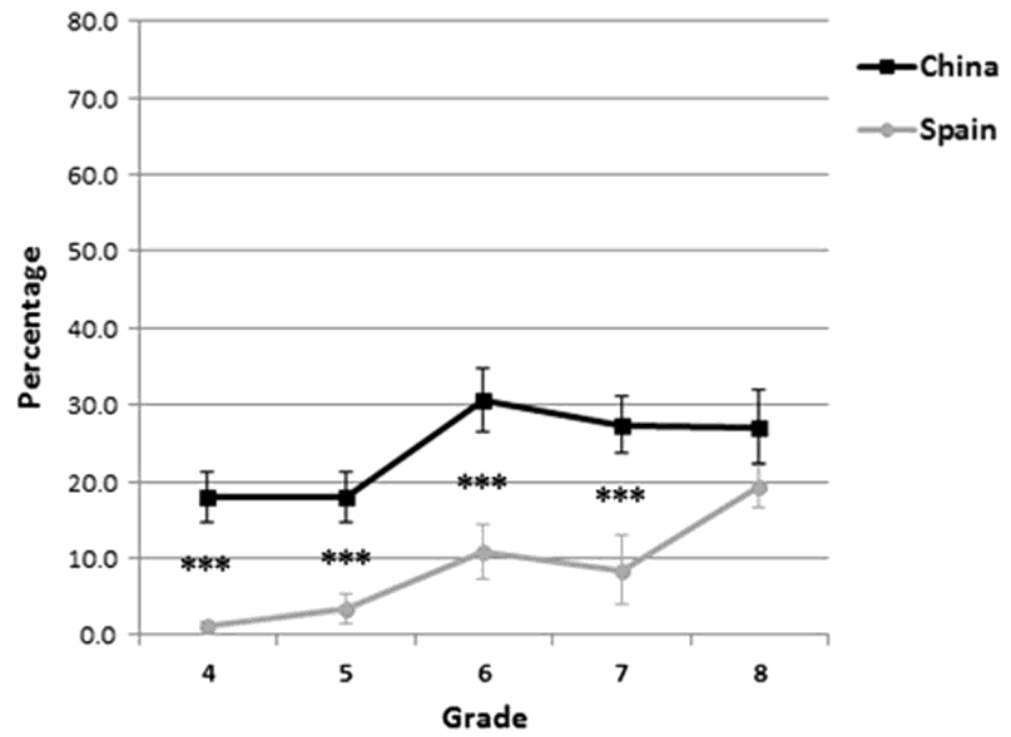

Fig. 4 The development of proportional methods on additive problems from 4th grade to 8th grade in China and Spain

"number structure" was significant, $X^{2}(1, N=925)=11.374, p=0.022$, and no other interaction effects that involved "number structure" were found. The analysis showed that Chinese and Spanish students used more additive methods in non-integer problems, independently the type of problem (additive or proportional). Further pairwise comparisons indicate that, for Chinese students, there was no significant difference of their additive answers between integer and noninteger problems in 4 th grade $(69.03$ vs. $72.59 \%)$ or 6th grade (47.91 vs. $50.00 \%)$, but such difference was significant in 5 th grade ( 59.27 vs. $66.86 \%)$, 7 th grade ( 42.80 vs. $53.76 \%$ ), and 8th grade (38.75 vs. $50.49 \%$ ). For Spanish students, the difference of additive answers to problems with different number ratios was not significant in 4th grade (50.39 vs. $53.46 \%)$, while such difference was significant in 5th grade (45.56 vs. $60.67 \%$ ), 6th grade (51.96 vs. 
$63.33 \%)$, 7 th grade (47.78 vs. $57.47 \%$ ), and 8th grade (45.68 vs. $56.18 \%$ ).

With regard to proportional methods, analysis showed that the main effect of "number structure" was significant, $X^{2}(1, N=925)=70.419, p<0.001$, and there was a significant

Table 2 Percentages of additive answers to additive and proportional problems with integer (I) or non-integer (N) ratios in China and Spain (\%)

\begin{tabular}{|c|c|c|c|c|c|c|c|c|}
\hline \multirow[t]{3}{*}{ Grade } & \multicolumn{4}{|l|}{ China } & \multicolumn{4}{|l|}{ Spain } \\
\hline & \multicolumn{2}{|c|}{ Additive problems } & \multicolumn{2}{|c|}{ Proportional problems } & \multicolumn{2}{|c|}{ Additive problems } & \multicolumn{2}{|c|}{ Proportional problems } \\
\hline & I & $\mathrm{N}$ & I & $\mathrm{N}$ & I & $\mathrm{N}$ & I & $\mathrm{N}$ \\
\hline 4 & 73.07 & 74.51 & 64.71 & 70.60 & 51.54 & 54.62 & 49.23 & 52.31 \\
\hline 5 & 61.50 & 70.50 & 57.00 & 63.01 & 43.37 & 62.50 & 47.79 & 58.82 \\
\hline 6 & 59.43 & 61.39 & 36.63 & 38.61 & 51.56 & 64.86 & 52.36 & 61.78 \\
\hline 7 & 52.22 & 63.34 & 33.88 & 43.88 & 47.58 & 58.87 & 47.98 & 56.05 \\
\hline 8 & 59.17 & 70.83 & 21.63 & 29.94 & 48.68 & 60.93 & 42.71 & 51.33 \\
\hline Total & 61.35 & 68.30 & 41.94 & 49.27 & 48.59 & 60.36 & 47.96 & 56.14 \\
\hline
\end{tabular}

Table 3 Percentages of proportional answers to proportional and additive problems with integer (I) or noninteger $(\mathrm{N})$ ratios in China and Spain $(\%)$

\begin{tabular}{|c|c|c|c|c|c|c|c|c|}
\hline \multirow[t]{3}{*}{ Grade } & \multicolumn{4}{|l|}{ China } & \multicolumn{4}{|l|}{ Spain } \\
\hline & \multicolumn{2}{|c|}{ Proportional problems } & \multicolumn{2}{|c|}{ Additive problems } & \multicolumn{2}{|c|}{ Proportional problems } & \multicolumn{2}{|c|}{ Additive problems } \\
\hline & I & $\mathrm{N}$ & I & $\mathrm{N}$ & I & $\mathrm{N}$ & I & $\mathrm{N}$ \\
\hline 4 & 26.80 & 20.14 & 21.16 & 15.02 & 10.03 & 0.49 & 5.87 & 0.24 \\
\hline 5 & 29.38 & 22.97 & 24.46 & 12.87 & 19.74 & 1.43 & 15.97 & 0.66 \\
\hline 6 & 52.42 & 53.03 & 29.74 & 31.63 & 21.77 & 6.09 & 20.15 & 5.52 \\
\hline 7 & 51.62 & 40.05 & 33.38 & 22.11 & 21.77 & 5.18 & 18.35 & 3.59 \\
\hline 8 & 67.91 & 54.59 & 32.92 & 22.07 & 33.27 & 19.68 & 25.99 & 14.05 \\
\hline Total & 45.16 & 36.88 & 28.07 & 19.98 & 20.26 & 3.49 & 15.84 & 2.20 \\
\hline
\end{tabular}

"grade" $\times$ "number structure" interaction effect, $X^{2}(1, N=925)=15.525, p=0.004$. Moreover, we found a significant "country" $\times$ "number structure" interaction effect, $x^{2}(4$, $\mathrm{N}=925)=32.167, \mathrm{p}<0.001$, and the interaction effect of "country" $\times$ "grade" $\times$ "number structure" was also significant, $X^{2}(4, N=925)=23.102, p<0.001$. Further analysis showed that for both countries, the interaction effect of "grade" $\times$ "number structure" was significant (Chinese, $X^{2}(4, N=453)=27.162, p<0.001$; Spanish, $\left.X^{2}(4, N=453)=20.911, p<0.001\right)$. Students in both countries used more proportional methods in integer problems, despite the type of problem (additive or proportional), and such a tendency was different in each grade. Pairwise comparisons showed that for Chinese students, number structure had significant effects on proportional answers in 4th grade (23.87 vs. $17.46 \%)$, 5th grade (26.86 vs. $17.35 \%)$, 7th grade (42.23 vs. $30.33 \%$ ), and 8th grade (50.46 vs. $36.85 \%)$. Interestingly, Chinese 6th graders were immune to the influence of number structure (40.62 vs. $41.91 \%$ ). For Spanish students who used more proportional methods on integer versions along 4th grade to 8 th grade, the proportional answers to problems with different number ratios were signif- 
icantly different in 4 th grade (7.67 vs. $0.35 \%)$, 5th grade (17.75 vs. $1.08 \%)$, 6th grade $(20.94$ vs. $5.81 \%)$, 7th grade (19.99 vs. $4.37 \%$ ), and 8th grade (29.51 vs. $16.67 \%$ ).

These results indicated that the number structure has an obvious impact on students' use of additive and proportional methods. Such influence seems to become stronger for the students of both countries along grades, though there were some exceptions (4th graders in China and Spain, 6th graders in China). Also, such an effect on the use of the proportional method is different in the two countries: for Chinese students, although the influence is strong in many grades, students' proportional responses to non-integer problems remained in a high level, and Chinese 6th graders are not affected. While for Spanish students, the impact of number structure is strong in all grades and the percentage of students' proportional answers to noninteger problems is low except in 8th grade.

\section{The effect of nature of quantities and its interaction with country}

Tables 4 and 5 present students' use of additive and proportional methods in addition and proportion problem with different nature of quantities along grade levels in China and Spain. For the use of additive methods, analysis results showed that the main effect of "nature of quantities" was not significant, $X^{2}(1, N=925)=0.026, p=0.872$, and no other interaction effects were found. 
Table 4 Percentages of additive answers to additive and proportional problems with discrete (D) or continuous (C) quantities in China and Spain (\%)

\begin{tabular}{|c|c|c|c|c|c|c|c|c|}
\hline \multirow[t]{3}{*}{ Grade } & \multicolumn{4}{|l|}{ China } & \multicolumn{4}{|l|}{ Spain } \\
\hline & \multicolumn{2}{|c|}{ Additive problems } & \multicolumn{2}{|c|}{ Proportional problems } & \multicolumn{2}{|c|}{ Additive problems } & \multicolumn{2}{|c|}{ Proportional problems } \\
\hline & $\mathrm{C}$ & $\mathrm{D}$ & $\mathrm{C}$ & $\mathrm{D}$ & $\mathrm{C}$ & $\mathrm{D}$ & $\mathrm{C}$ & $\mathrm{D}$ \\
\hline 4 & 73.57 & 74.02 & 67.76 & 67.69 & 50.00 & 56.15 & 51.54 & 50.00 \\
\hline 5 & 66.67 & 65.62 & 58.53 & 61.55 & 53.68 & 52.21 & 55.15 & 51.47 \\
\hline 6 & 59.42 & 61.39 & 37.61 & 37.62 & 59.38 & 57.03 & 60.94 & 53.13 \\
\hline 7 & 58.44 & 57.31 & 38.23 & 39.31 & 55.24 & 51.21 & 51.61 & 52.42 \\
\hline 8 & 64.40 & 66.00 & 23.97 & 27.26 & 54.97 & 54.64 & 46.36 & 47.68 \\
\hline Total & 64.60 & 65.01 & 44.80 & 46.51 & 54.67 & 54.26 & 53.15 & 50.94 \\
\hline
\end{tabular}

For the use of proportional methods, the main effect of "nature of quantities" was significant $X^{2}(1, N=925)=8.895, p=0.003$, and there is also a significant "country" $\times$ "nature of quantities" interaction effect, $X^{2}(1, N=925)=10.131, p=0.001$. Pairwise comparisons indicated that for Chinese students, the difference of their use of proportional methods on the problems with different quantities was not significant (31.82 vs. $31.68 \%, p=0.877$ ), while for Spanish students, such difference was significant. Students applied slightly less proportional approaches on problems with continuous quantities compared to discrete problems ( 6.31 vs. $8.47 \%, p=0.003$ ) in two types of problems. No other relevant interaction effects were found.

\section{Discussion and conclusions}

The aim of this study was to investigate the 4th to 8th grade students' development of their additive and proportional reasoning in solving two types of missing-value problems from a cross-national perspective.

Table 5 Percentages of proportional answers to proportional and additive problems with discrete (D) or continuous (C) quantities in China and Spain (\%)

\begin{tabular}{|c|c|c|c|c|c|c|c|c|}
\hline \multirow[t]{3}{*}{ Grade } & \multicolumn{4}{|l|}{ China } & \multicolumn{4}{|l|}{ Spain } \\
\hline & \multicolumn{2}{|c|}{ Proportional problems } & \multicolumn{2}{|c|}{ Additive problems } & \multicolumn{2}{|c|}{ Proportional problems } & \multicolumn{2}{|c|}{ Additive problem } \\
\hline & $\mathrm{C}$ & $\mathrm{D}$ & $\mathrm{C}$ & $\mathrm{D}$ & $\mathrm{C}$ & $\mathrm{D}$ & $\mathrm{C}$ & $\mathrm{D}$ \\
\hline 4 & 22.17 & 24.45 & 18.63 & 17.65 & 51.54 & 50.00 & 3.08 & 3.08 \\
\hline 5 & 27.64 & 24.49 & 18.00 & 19.50 & 55.15 & 51.47 & 7.35 & 9.56 \\
\hline 6 & 52.48 & 52.97 & 31.19 & 30.20 & 60.94 & 53.13 & 11.72 & 14.06 \\
\hline 7 & 47.73 & 43.83 & 26.11 & 29.44 & 51.61 & 52.42 & 9.68 & 12.50 \\
\hline 8 & 60.19 & 62.81 & 30.00 & 25.00 & 46.36 & 47.68 & 19.54 & 20.53 \\
\hline Total & 41.28 & 40.77 & 24.34 & 23.98 & 53.15 & 50.94 & 8.86 & 10.32 \\
\hline
\end{tabular}


We found that Spanish students and Chinese students had similarities related to the developmental trends in additive and proportional reasoning. For both China and Spain, students' correct responses to proportion problems increased along grades. However, students' additive reasoning remains in a stable level from 4th to 8th grade for Spanish students but decreases between 4th and 7th grade in China.

Considering the overuse of additive and proportional methods, there were also some similarities between Chinese and Spanish students. In both countries, lower-grade students tend to apply additive methods for all kind of problems, while higher-grade students prefer to use proportional methods more often. Such a tendency indicated that although many students acquired additive or proportional knowledge, they were not good mathematical reasoners because they could neither recognize problems correctly nor use the proper strategy. Such cross-national results are consistent with previous studies (Fernández et al. 2011; Li et al. 2014; Van Dooren et al. 2009).

We also found differences between the two countries. First, the percentages of Chinese students' correct answers to proportion problems increased with grade level; in the meantime, the percentages of using the additive method in proportion problems decreased with grade level, but there was also a decrease in the use of this method in addition problems until 7th grade. This result showed that Chinese students' proportion problem solving improved significantly with grade level, from elementary school to junior high school, but this does not happen with addition problems. Spanish students' use of proportional methods also increased with grade level, but this increase was less significant than the increase seen with Chinese students. However, Spanish students' additive reasoning remains stable during these grades.

Second, there are also some differences of the overuse of additive and proportional methods between Spanish and Chinese students. For Spanish students, from grade 4 to grade 8, they consistently showed a tendency to overuse the additive method and this tendency starts to slightly decrease from 6th grade onward; however, for Chinese students, 4th and 5th graders greatly overused the additive method, but this tendency decreased significantly from 6th grade onward when the overuse of proportional method began to increase. Comparatively, Spanish students started to show great overuse tendency of proportionality in 7 th grade, whereas there was a decrease in the correct use of the additive method in addition problems. However, Chinese students showed a different developmental model that is inconsistent with previous studies, which suggests that students would apply additive methods blindly in the medium grades in elementary school and after they entered high grades in elementary school, they started to used proportional method without considering the situations (Fernández et al. 2012; Van Dooren et al. 2010). For Chinese students, the overuse of proportional methods started to slightly decrease from 6th grade onward; moreover, students started to apply more additive method on addition problems.

We believe that there could be many reasons for these different trends. On the one hand, the development from additive to proportional reasoning occurs at a different age in China and Spain because the curriculum of proportionality comes earlier in the Chinese context. Chinese 6th graders had already started to learn the direct and inverse proportionality and the "rule of three", which is critical for students to solve proportion problems in a missing-value format; Spanish students do not learn these concepts and methods until 7th grade. On the other hand, it is also because of the different educational environments in China and Spain. First, Chinese math curricula requirements are more difficult than those of western countries and this could explain why Chinese students outperform their western peers. For example, a Chinese study indicated that although Shanghai 
students get high PISA scores, the test is considered too easy for 15-year-old students in China. The researchers found that more than $75 \%$ of 6th graders can correctly answer $60 \%$ or more math items in the PISA test. Moreover, more than $73 \%$ of elementary school teachers believe that the difficulty of most test items are lower than the 6th grade level (Ding and Zhou 2014). In this study, the missing-value problem may be easy for Chinese secondary students because they learned about it in elementary school. As a result, they performed better than the Spanish students. Second, Chinese students spend more time on learning. They usually devote more time to homework, and their teachers also place a great emphasis on mathematics homework (Leung 2014; Siegler and Mu 2008). Dello-Iacovo (2009) also pointed out that Chinese students get extra training and practice by attending a lot of math cram classes. Such repetitive learning may also improve their problemsolving skills.

Interestingly, previous studies also indicated that Chinese students are not necessarily better performers on non-routine problem solving (Cai 2000; Cai and Nie 2007), and this may explain why Chinese students overused additive and proportional methods just as the Spanish students did in this study that is, proportion problems for students in lower grades and addition problems for students in higher grades would, in both cases, be considered unconventional.

In addition to cultural factors, this study also examined the effects of number structure and nature of quantities on students' additive and proportional reasoning. We found that both Chinese students and Spanish students were inclined to use more proportional methods with an integer ratio, independent of the type of problem. This result confirms the findings of previous studies and indicates that students have a tendency to overlook the nature of the problem (additive or proportional) and to rely on the characteristic of the numbers (ratios between numbers) to solve problems in hand (Fernández et al. 2011, 2012; Van Dooren et al. 2009).

With regard to proportional methods, we also found that the influence of number structure interacted with national differences: for Chinese students, the influence of number structure is significant and strong in all the grades except 6th grade; for Spanish students, such influence is strong in every grade, and from 4th grade to 7th grade, the percentage of students' proportional answers to non-integer problems showed a floor effect. There are some explanations for such differences. First, Chinese students' proportional problem-solving skills are better than their Spanish peers, thus their proportional responses to problems remained at a high level even with a non-integer ratio. Second, Chinese 6th graders were reaching their peak understanding of using proportional methods; and during the learning of proportionality, teachers in China usually emphasize the concept of unit quantity (how many quantities). When the Chinese students got such a problem, they tended to rely on "unit quantity thinking" in which a fragile (non-integer) discrete quantity is still meaningful (e.g., Mike loaded 7.5 boxes per hour). This may explain why they are less vulnerable to number structures when using proportional methods and thus became outliers in our whole sample.

Considering the effect of nature of quantities, we found that Chinese students were not affected by this variable, and Spanish students used more proportional methods when the numbers were discrete quantities; however, this effect was very small. We believe that the absence of the effect and the low effect of this variable might be due to the missing-value format used in the study. Previous studies observed a stronger effect by using a comparison format (Boyer et al. 2008; Jeong et al. 2007) or by using visual stimuli (Spinillo and Bryant 1999). Furthermore, the values of quantities are numbers in a word format problem and they are difficult to be "visualized" as continuous or discrete during the operation (Fernández et al. 2012). Thus, to identify the effect of nature of quantities, further research should avoid these limitations and 
use a new form of problem with visual materials (discrete squares or continuous column) instead of word problems.

In general, we found some common developmental trends of additive and proportional reasoning of students from China and Spain, and many students of both countries overuse additive and proportional methods. Meanwhile, we also found that Chinese students and Spanish students had different profiles on the same math items. This suggests that a crossnational study is necessary and that this kind of study would give us a chance to understand the developmental trajectory of the mathematical thinking of students in different countries, as well as to explore how mathematics education practices affect students' performance. Still, we have to be very discreet when generalizing the conclusion in consideration of the following limitations in the present study. First, we present a special format of a missing-value problem. These problems share a very similar structure and participants may use the same method to solve the problems even when they are confronted with different items. Previous studies demonstrated that such a characteristic may suppress genuine problem-solving processes (Wüstenberg et al. 2012) and thus induce the overuse of additive or proportional methods. Future studies should use a multiple-item test with different types of problems to examine whether these results can be replicated or not. Second, the samples of the two countries were not fully comparable. Since we used an age-match in this study and we did not control for students' intelligence or math abilities, national differences could not fully account for the different results in China and Spain. Therefore, an ability-match design may be necessary because in an age-match design the difference between two groups in mathematical thinking might be interpreted in terms of a relative developmental delay in the acquisition of mathematics knowledge and skills and not in differences in thinking (Muldoon et al. 2011). Third, this is a cross-sectional study and strictly speaking, we could only observe the difference between different grades but not the developmental trajectory of students' mathematical thinking. Future research may adopt a longitudinal design to answer this question.

Finally, we also want to present some theoretical and educational implications. For further studies considering a cross-national (or cross-cultural) perspective, we suggest that more participant countries should be involved. Many reports of cross-national comparative studies focus on comparisons between high-performing countries and lowperforming countries; however, Jiang et al. (2014) suggest that the comparisons between two high-performing countries will provide us an opportunity to understand the strengths and weaknesses of mathematics education more precisely, and even when two groups of students appear to perform similarly on a set of mathematical tasks, their underlying thinking may be quite different. Therefore, it would be interesting to focus on both low-high-performing comparisons and high-high-performing comparisons. Moreover, the participants in our study are students from 4th grade to 8th grade. However, the length of schooling may differ in different countries. Thus, further research may consider a broader age range of participants (e.g., high school students or adults) and examine the developmental models more precisely.

In view of students' tendency to overuse additive proportional methods in both countries, it is necessary for teachers to emphasize the nature of different mathematical structures during classroom teaching in order to increase students' understanding and eliminate the misleading superficial characteristics. For example, teachers could present additive and proportion problems in a missing-value format at the same time and explain the underlying relationships instead of teaching the skills only. This would help students learn how to discern different problem situations and then to choose the 
proper solutions (Kaput and West 1994). Moreover, a previous study had shown that fostering a higher-order thinking skill (e.g., a complex problem-solving skill or induc- tive reasoning) may also improve students' domain-specific problem solving (Molnár et al. 2013). Therefore, explicit training in additional ways to develop higher-order thinking skills would be helpful to improve students' mathematical thinking and problems-solving skills. Meanwhile, Neo-Piagetian model suggested that students make errors in solving problems not only because they lack the cognitive ability but also because they fail to inhibit an overlearned strategy (Siegler 1999; Houdé 2007; Lubin et al. 2013). Therefore, cognitive training including inhibitory control and meta- cognition may be necessary for low-performing students.

\section{References}

Beijing Normal University Press. (2013). Compulsory education curriculum standard experimental textbook mathematics [义务教育课程标准实验教科书数学]. Beijing: Beijing Normal University Press.

Boyer, T. W., Levine, S. C., \& Huttenlocher, J. (2008). Development of proportional reasoning: where young children go wrong. Developmental Psychology, 44(5), 1478.

Cai, J. (2000). Mathematical thinking involved in US and Chinese students' solving of process-constrained and process-open problems. Mathematical Thinking and Learning, 2(4), 309-340.

Cai, J. (2004). Why do US and Chinese students think differently in mathematical problem solving?: impact of early algebra learning and teachers' beliefs. The Journal of Mathematical Behavior, 23(2), 135-167.

Cai, J., \& Nie, B. (2007). Problem solving in Chinese mathematics education: research and practice. ZDM, 39(56), 459-473.

DECRETO 108/2014, de 4 de julio, del Consell, por el que establece el currículo y desarrolla la ordenación general de la educación primaria en la Comunitat Valenciana. [2014/6347].

DECRETO 112/2007, de 20 de julio, del Consell, por el que se establece el currículo de la Educación Secundaria Obligatoria en la Comunitat Valenciana. [2007/9717].

De Bock, D., Van Dooren, W., Janssens, D., Verschaffel, L. (2007). The illusion of linearity: from analysis to improvement (Vol. 41): Springer Science \& Business Media.

Dello-Iacovo, B. (2009). Curriculum reform and 'quality education' in China: an overview. International Journal of Educational Development, 29(3), 241-249.

Ding, D., \& Zhou, J. (2014). How difficult the PISA mathematics test is: a third explaination to Shanghai students' PISA performance according to an investigation in two Beijing elementary schools. Administration in Elementary and Secondary School [中小学管理], (10), 24-27.

Fan, L., Wong, N.-Y., Cai, J., Li, S. (2004). How Chinese learn mathematics: perspectives from insiders (Vol. 1): World Scientific.

Fernández, C., \& Llinares, S. (2012). Características del desarrollo del razonamiento proporcional en la educación primaria y secundaria. Enseñanza de las Ciencias, 30(1), 0129-0142.

Fernández, C., Llinares, S., Van Dooren, W., De Bock, D., Verschaffel, L. Effect of the number structure and the quantity nature on secondary school students' proportional reasoning. In M. Tzekaki, M. Kaldrimidou, \& H. Sakonidis (Eds.), Proceedings of the 33rd Conference of the International Group for the Psychology of Mathematics Education., 2009 (Vol. 3, pp. 25-32): PME

Fernández, C., Llinares, S., Van Dooren, W., De Bock, D., \& Verschaffel, L. (2011). Effect of number structure and nature of quantities on secondary school students' proportional reasoning. Studia Psychologica, 53(1), 69-81.

Fernández, C., Llinares, S., Van Dooren, W., De Bock, D., \& Verschaffel, L. (2012). The development of students' use of additive and proportional methods along primary and secondary school. European Journal of Psychology of Education, 27(3), 421-438.

Harel, G., \& Confrey, J. (1994). The development of multiplicative reasoning in the learning of mathematics: Luce Irigaray and 'the Greeks'. New York: SUNY Press.

Hart, K. M., Brown, M. L., Kuchemann, D. E., Kerslake, D., Ruddock, G., \& McCartney, M. (1981). Children's understanding of mathematics: 11-16. London: John Murray.

Houdé, O. (2007). First insights on "neuropedagogy of reasoning". Thinking and Reasoning, 13(2), 81-89.

Jeong, Y., Levine, S. C., \& Huttenlocher, J. (2007). The development of proportional reasoning: effect of continuous versus discrete quantities. Journal of Cognition and Development, 8(2), 237-256.

Jiang, C., Hwang, S., \& Cai, J. (2014). Chinese and Singaporean sixth-grade students' strategies for solving problems about speed. Educational Studies in Mathematics, 87(1), 27-50.

Kaput, J., \& West, M. M. (1994). Missing value proportional reasoning problems: factors affecting informal reasoning 
patterns. In G. Harel, \& J. Confrey (Eds.), The Development of Multiplicative Reasoning in the Learning of Mathematics: Luce Irigaray and 'the Greeks'. New York: SUNY Press \%\% 2016-03-01 14:43:00.

Leung, F. K. (2014). What can and should we learn from international studies of mathematics achievement? Mathematics Education Research Journal, 26(3), 579-605.

Li, X.. Jiang, R., Qian, Y. (2014). 5 8 graders' overuse of proportionality on missing-value problems. Journal of Mathematics Education [数学教育学报], (06), 73-77.

Lubin, A., Vidal, J., Lanoë, C., Houdé, O., \& Borst, G. (2013). Inhibitory control is needed for the resolution of arithmetic word problems: a developmental negative priming study. Journal of Educational Psychology, 105(3), 701.

Misailidou, C., \& Williams, J. (2003). Diagnostic assessment of children's proportional reasoning. The Journal of Mathematical Behavior, 22(3), 335-368.

Molnár, G., Greiff, S., \& Csapó, B. (2013). Inductive reasoning, domain specific and complex problem solving: relations and development. Thinking Skills and Creativity, 9, 35-45.

Muldoon, K., Simms, V., Towse, J., Menzies, V., \& Yue, G. (2011). Cross-cultural comparisons of 5-year-olds' estimating and mathematical ability. Journal of Cross-Cultural Psychology, 42(4), 669-681.

Mullis, I. V., Martin, M., Foy, P., Olson, J., Preuschoff, C., \& Erberber, E. (2008). TIMSS 2007 International Mathematics Report (Chestnut Hill, MA: TIMSS \& PIRLS International Study Center, Boston College). Chestnut Hill: TIMSS \& PIRLS International Study Center, Boston College.

Mullis, I. V., Martin, M. O., Foy, P., Arora, A. (2012). TIMSS 2011 international results in mathematics. Herengracht 487, Amsterdam, 1017 BT, The Netherlands.: ERIC.

Nesher, P. (1988). Multiplicative school word problems: theoretical approaches and empirical findings. Number Concepts and Operations in the Middle Grades, 2, 19-40.

OECD (2010). PISA 2009 results: what students know and can do: student performance in reading, mathematics and science (Vol. 1): OECD.

Robitaille, D. F., \& Travers, K. J. (1992). International studies of achievement in mathematics. In D. A Grouws (Ed.), Handbook for research on mathematics and teaching (pp. 687-709). New York: Macmillan.

Siegler, R. S. (1999). Strategic development. Trends in Cognitive Sciences, 3(11), 430-435.

Siegler, R. S., \& Mu, Y. (2008). Chinese children excel on novel mathematics problems even before elementary school. Psychological Science, 19(8), 759-763.

Spinillo, A. G., \& Bryant, P. E. (1999). Proportional reasoning in young children: part-part comparisons about continuous and discontinuous quantity. Mathematical Cognition, 5(2), 181-197.

Tourniaire, F., \& Pulos, S. (1985). Proportional reasoning: a review of the literature. Educational Studies in Mathematics, 16(2), 181-204.

Van Dooren, W., De Bock, D., Evers, M., Verschaffel, L. (2009). Students' overuse of proportionality on missing-value problems: how numbers may change solutions. Journal for Research in Mathematics Education, 187-211.

Van Dooren, W., De Bock, D., Janssens, D., Verschaffel, L. (2008). The linear imperative: an inventory and conceptual analysis of students' overuse of linearity. Journal for Research in Mathematics Education, 311342.

Van Dooren, W., De Bock, D., Vleugels, K., \& Verschaffel, L. (2010). Just answering... or thinking? Contrasting pupils' solutions and classifications of missing-value word problems. Mathematical Thinking and Learning, 12(1), 20-35.

Verschaffel, L., Greer, B., \& De Corte, E. (2007). Whole number concepts and operations. In F. K. Lester (Ed.), Second handbook of research on mathematics teaching and learning (pp. 557-628). Greenwich: Information Age Publishing.

Wüstenberg, S., Greiff, S., \& Funke, J. (2012). Complex problem solving-more than reasoning? Intelligence, 40(1), 1-14. 